\title{
BMJ Open The association between a non-invasive hepatic fibrosis score and urolithiasis among non-alcoholic fatty liver disease (NAFLD) patients in China: a cross- sectional study
}

Shaoyou Qin, ${ }^{1}$ Jiangbin Wang, ${ }^{1}$ Changyu Zhou, ${ }^{1}$ Yonggui Zhang, ${ }^{1}$ Yan $\mathrm{Xu},{ }^{1}$ Xu Wang, ${ }^{1}$ Song Wang ${ }^{\circledR 2}$

To cite: Qin S, Wang J, Zhou C, et al. The association between a non-invasive hepatic fibrosis score and urolithiasis among non-alcoholic fatty liver disease (NAFLD) patients in China: a cross-sectional study. BMJ Open 2019;9:e027702. doi:10.1136/ bmjopen-2018-027702

- Prepublication history for this paper is available online. To view these files, please visit the journal online (http://dx.doi. org/10.1136/bmjopen-2018027702).

Received 04 November 2018 Revised 23 June 2019 Accepted 23 July 2019
Check for updates

(C) Author(s) (or their employer(s)) 2019. Re-use permitted under CC BY-NC. No commercial re-use. See rights and permissions. Published by BMJ.

${ }^{1}$ Department of Gastroenterology and Hepatology, China-Japan Union Hospital of Jilin University, Changchun, China

${ }^{2}$ Department of Urology, the First Hospital of Jilin University, Changchun, China

Correspondence to Dr Song Wang;

wangsongurologist@163.com

\section{ABSTRACT}

Objective Mounting data now support a strong link between the presence of non-alcoholic fatty liver disease (NAFLD) and an increased risk of urolithiasis. However, little is known on the association between hepatic fibrosis and the risk of urolithiasis among NAFLD patients. Therefore, this study aimed to investigate the prevalence of urolithiasis among NAFLD patients and determine whether the Fibrosis-4 (FIB-4) score, a surrogate marker of hepatic fibrosis, is associated with urolithiasis among NAFLD patients.

Design Cross-sectional studies.

Setting China.

Methods A total of 2058 adult patients with NAFLD were included in this study. Logistic regression analysis was used to detect the association between FIB-4 score and urolithiasis. Receiver operating characteristic (ROC) curve analysis was used to assess the diagnostic value of FIB-4 score for the detection of urolithiasis among NAFLD patients. Results $200(9.7 \%)$ individuals had ultrasonographydiagnosed urolithiasis among 2058 NAFLD patients. FIB-4 score $(\mathrm{OR}=1.58 ; 95 \% \mathrm{Cl} 1.06$ to 2.31), age $(\mathrm{OR}=1.11$; $95 \% \mathrm{Cl} 1.08$ to 1.13$)$, obesity ( $\mathrm{OR}=3.16 ; 95 \% \mathrm{Cl} 2.29$ to 4.39) and hyperuricemia ( $\mathrm{OR}=3.79 ; 95 \% \mathrm{Cl} 2.67$ to 5.36$)$ were independent factors associated with urolithiasis among NAFLD patients. Moreover, a novel algorithm including multiple variables (FIB-4 score, age, obesity and hyperuricemia) showed an area under a ROC curve of 0.813 (95\% Cl 0.795 to 0.829 ) for identifying urolithiasis among NAFLD patients. The optimal cut-off value of $>$ -2.23 for the multivariate model provides a sensitivity of $76 \%$ and a specificity of $74 \%$ for predicting urolithiasis among NAFLD patients.

Conclusion Urolithiasis among NAFLD patients is associated with FIB-4 score. Further, a novel algorithm based on FIB-4 score could serve as a useful tool for identifying individuals with a higher risk of urolithiasis among NAFLD patients, although prospective cohort studies are still needed in the future.

\section{INTRODUCTION}

Non-alcoholic fatty liver disease (NAFLD) is the most common cause of chronic liver
Strengths and limitations of this study

- A novel algorithm based on non-invasive clinical and biochemical parameters was established for identifying individuals at high risk for urolithiasis among non-alcoholicfatty liver disease (NAFLD) patients.

- Multiple potential confounders were adjusted in the logistical regression model to determine factors associated with urolithiasis among NAFLD.

- The causal association between the non-invasive hepatic fibrosis score and urolithiasis among NAFLD patients could not be established, as the cross-sectional design of this study.

disease among adults globally. NAFLD comprises a disease spectrum ranging from simple steatosis to progressive non-alcoholic steatohepatitis characterised by inflammation, necrosis and fibrosis, which is at high risk of progressing to cirrhosis and hepatocellular carcinoma. ${ }^{12}$ The estimated prevalence of NAFLD ranges from $17 \%$ to $33 \%$ in the general population in various countries and is continuing to increase, due to the global epidemics of metabolic syndrome. ${ }^{3}$

Recently, the increasing recognition of the importance of NAFLD and its close link with the metabolic syndrome has stimulated an interest in the potential role of NAFLD in the development and progression of extrahepatic diseases including chronic kidney disease (CKD) and urolithiasis. ${ }^{4-6}$ Numerous studies provide evidence of a strong association between the risk of prevalent CKD and the presence and fibrosis stage of NAFLD (measured with either biopsy or FIB-4 score as a non-invasive measure for hepatic fibrosis) ${ }^{7-13}$ Similarly, the putative association between NAFLD and urolithiasis has also attracted scientific interest. A cross-sectional 


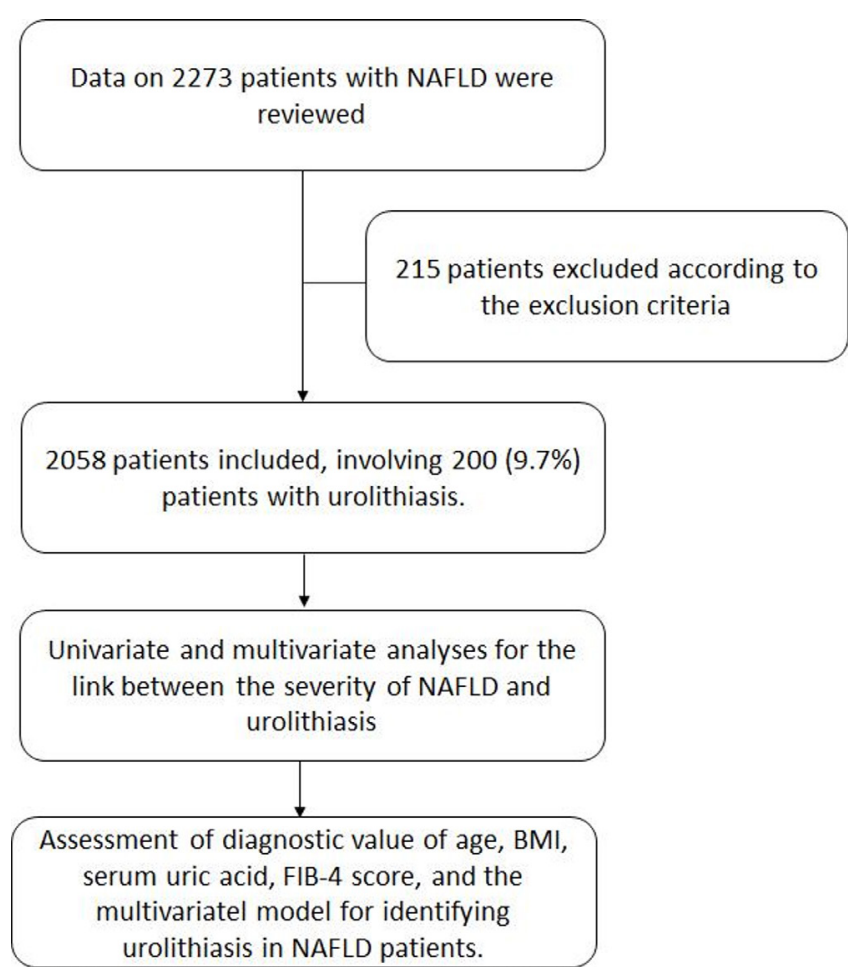

Figure 1 Study flow diagram. BMI, body mass index; NAFLD, non-alcoholic fatty liver disease.

study involving 3719 Chinese men suggested that NAFLD was related to a higher prevalence of urinary calculi. Again, a meta-analysis involving seven observational studies and 226541 individuals demonstrated a 1.73-fold increased risk of urolithiasis among NAFLD patients compared with healthy controls. ${ }^{14}$ In total, NAFLD has been increasingly regarded as a critical risk factor for both CKD and urolithiasis.

Although there is accumulating evidence that the presence of NAFLD is closely associated with urolithiasis, the available data on the association between hepatic fibrosis and urolithiasis are quantitatively limited, partly because it is not appropriate to perform a liver biopsy in large epidemiological studies. The impact of hepatic fibrosis on the risk of urolithiasis deserves particular attention, given the potential implications for screening strategies in the increasing number of individuals with NAFLD. Preliminary study has demonstrated that the FIB-4 score was a surrogate marker of hepatic fibrosis, providing high diagnostic accuracy for advanced fibrosis with an area under a ROC curve (AUROC) of $0.86 .{ }^{15}$ The aim of this cross-sectional study, thus, was to investigate the prevalence of urolithiasis among NAFLD patients and determine whether the FIB-4 score is associated with urolithiasis among NAFLD patients.

\section{METHOD}

\section{Study design}

A total of 2273 ultrasonography-diagnosed NAFLD patients who underwent a comprehensive medical examination in China-Japan union hospital from January 2015 to December 2017 were initially eligible. The exclusion criteria included: (1) Patients with secondary causes of chronic liver disease (alcohol abuse, viral hepatitis, medications, autoimmune hepatitis). (2) Patients who did not undergo urinary tract ultrasounds as part of the examination. (3) Patients who had missing data. By the exclusion criteria, 215 patients were excluded, and ultimately, the remaining 2058 individuals with NAFLD were included. Basic demographic data of the study participants were obtained from the medical records of patients. Results were analysed anonymously. The medical history (including diabetes, hypertension) and lifestyle habits (smoking) of each participant were taken from a self-report questionnaire issued prior to the medical examination. Due to the retrospective nature of the study, written informed consent was waived. Patient information can be sufficiently anonymised.

\section{Ascertainment of NAFLD and urolithiasis}

Abdominal ultrasound for the detection of fatty liver and urolithiasis was carried out by registered medical sonographers who were blinded to the subjects' data. In accordance with the practice guideline by the American Association for the Study of Liver Diseases, NAFLD was diagnosed by liver ultrasound that revealed a bright liver and a diffusely echogenic change in the liver parenchyma. ${ }^{16}$ Renal calculi were diagnosed by urinary tract ultrasonography revealing curvilinear, echogenic foci with posterior acoustic shadowing. ${ }^{17}$

\section{Clinical and laboratory data collection}

The body mass index (BMI) was calculated using the following formula: weight in kilograms / (height in metres $)^{2} \quad\left(\mathrm{~kg} / \mathrm{m}^{2}\right)$. Waist circumference $(\mathrm{cm})$ was measured midway between the lower costal margin and the iliac crest at the end of a normal expiration of breath by a well-trained nurse. Blood pressure was measured on the right arm after $\mathrm{a} \geq 10 \mathrm{~min}$ rest using an electronic manometer. Early morning blood samples were taken from each patient after overnight fasting and subsequently analysed in the central certified laboratory of the China-Japan union hospital. The level of alanine aminotransferase (ALT), aspartate aminotransferase (AST), gamma-glutamyl transpeptidase, fasting plasma glucose, serum uric acid, creatinine, fasting total cholesterol, triglycerides, low-density lipoprotein cholesterol and high-density lipoprotein cholesterol was measured.

Obesity was defined as a BMI of $25 \mathrm{~kg} / \mathrm{m}^{2}$ and above. ${ }^{18}$ Diabetes mellitus was identified as a fasting plasma glucose concentration of $126 \mathrm{mg} / \mathrm{dL}$ and above, or a self-reported history of diabetes mellitus, or treatment of dietary modification, or the use of antidiabetic medication. Hyperuricemia was defined as a serum uric acid level of $>6.8 \mathrm{mg}$ / $\mathrm{dL}$ for men or $6 \mathrm{mg} / \mathrm{dL}$ for women. Hypertension was defined as a systolic pressure of at least $130 \mathrm{~mm} \mathrm{Hg}$, or a diastolic pressure of at least $85 \mathrm{~mm} \mathrm{Hg}$, or the use of antihypertensive agents. Dyslipidemia was defined as total cholesterol of $\geq 240 \mathrm{mg} / \mathrm{dL}$ or use of specific medication. 
Table 1 Clinical characteristics of the study population with NAFLD by urolithiasis status

\begin{tabular}{|c|c|c|c|c|}
\hline & & Urolithiasis am & LD patients & \\
\hline & Overall & No & Yes & $P$ value \\
\hline Age (years) & $53(46,60)$ & $52(46,59)$ & $61(55,68)$ & $<0.01^{*}$ \\
\hline Female & $872(0.42)$ & $794(0.43)$ & $78(0.39)$ & \\
\hline Male & $1186(0.58)$ & $1064(0.57)$ & $122(0.61)$ & \\
\hline No & $1684(0.82)$ & $1520(0.82)$ & $164(0.82)$ & \\
\hline Yes & $374(0.18)$ & $338(0.18)$ & $36(0.18)$ & \\
\hline Hypertension & & & & 0.731 \\
\hline No & $1660(0.81)$ & $1501(0.81)$ & $159(0.8)$ & \\
\hline Yes & $398(0.19)$ & $357(0.19)$ & $41(0.2)$ & \\
\hline Yes & $224(0.11)$ & $202(0.11)$ & $22(0.11)$ & \\
\hline BMI & $26.27 \pm 3.7$ & $26.14 \pm 3.7$ & $27.43 \pm 3.51$ & $<0.01^{\star}$ \\
\hline Obesity & & & & $<0.01^{\star}$ \\
\hline No & $1274(0.62)$ & $1192(0.64)$ & $82(0.41)$ & \\
\hline Yes & $784(0.38)$ & $666(0.36)$ & $118(0.59)$ & \\
\hline AST (IU/L) & $47 \pm 7.09$ & $46.92 \pm 7.11$ & $47.8 \pm 6.82$ & 0.083 \\
\hline ALT (IU/L) & $60.42 \pm 8.99$ & $60.56 \pm 8.98$ & $59.09 \pm 9.02$ & $0.03^{*}$ \\
\hline GGT (IU/L) & $70.83 \pm 8.96$ & $70.73 \pm 8.91$ & $71.75 \pm 9.44$ & 0.143 \\
\hline $\operatorname{PLT}\left(\times 10^{9} / \mathrm{L}\right)$ & $249 \pm 44$ & $249 \pm 44$ & $248 \pm 46$ & 0.816 \\
\hline Hyperuricemia & & & & $<0.01^{*}$ \\
\hline No & $1671(0.81)$ & $1545(0.83)$ & $126(0.63)$ & \\
\hline Yes & $387(0.19)$ & $313(0.17)$ & $74(0.37)$ & \\
\hline FIB-4 score & $1.28(1.04,1.6)$ & $1.25(1.02,1.57)$ & $1.57(1.31,1.78)$ & $<0.01^{*}$ \\
\hline
\end{tabular}

*Statistically significant difference.

ALT, alanine aminotransferase; AST, aspartate aminotransferase; BMI, body mass index; GGT, gamma-glutamyl transpeptidase; HDL, highdensity lipoprotein; NAFLD, non-alcoholic fatty liver disease; NS, no significant difference; PLT, platelet; TG, triglyceride.

\section{Model calculations}

FIB-4 score was calculated and used to evaluate the hepatic fibrosis non-invasively. The formulas for FIB-4 score was as follows: FIB- 4 score $=$ age $($ years $) \times$ AST $($ IU $/ \mathrm{L})$ $/\left(\right.$ platelet count $\left(10^{9} / \mathrm{L}\right) \times(\operatorname{ALT}(\mathrm{IU} / \mathrm{L}))^{1 / 2}$.

\section{Patients and public involvement}

Patients and/or the general public were not involved in this study.

\section{Statistical analysis}

Continuous variables were expressed as the mean $\pm \mathrm{SD}$ and categorical variables as frequencies with percentages.
Comparisons were performed using the $\chi^{2}$ test or Fisher's exact test for categorical variables and the independent Student's t-test or Mann-Whitney U test for numerous variables. Multivariate analysis was performed using a logistic regression model to determine the association between the FIB-4 score and urolithiasis among individuals with NAFLD. Covariates in the multivariable model, which included age, sex, obesity, diabetes mellitus, hypertension, hyperlipidemia and current smoking, were chosen based on their clinical importance as well as statistical significance. The diagnostic value of FIB-4 score, as well as other independently associated factors for discerning 
urolithiasis, was subsequently evaluated using receiver operating characteristic (ROC) curves analysis.

Moreover, the AUROC of these independent factors of urolithiasis among NAFLD patients taken together was assessed using the multivariable logistic regression model. The optimal cut-off values were calculated by maximising the sum of sensitivity +specificity. The comparison for the value of AUROC was performed using a z-test according to the method of DeLong et al. ${ }^{19}$ A two-tailed $\mathrm{p}$ value $<0.05$ was considered statistically significant. All statistical analysis was performed using medcalc and $\mathrm{R}$ package for windows. ${ }^{20}$

\section{RESULTS}

\section{Demographic characteristics of the participants}

A total of 2273 patients diagnosed as NAFLD were initially included. Two hundred and fifteen patients were excluded according to the exclusion criteria for a total of 2058 patients for inclusion in the study (figure 1), with a median age of $53(46,60)$ years and a male predominance ( $58 \%$ vs $42 \%)$. The demographic, clinical characteristics of the studied population with and without urolithiasis were shown in table 1 . Of the study participants, 200 (9.7\%) individuals had ultrasonography-diagnosed urolithiasis.
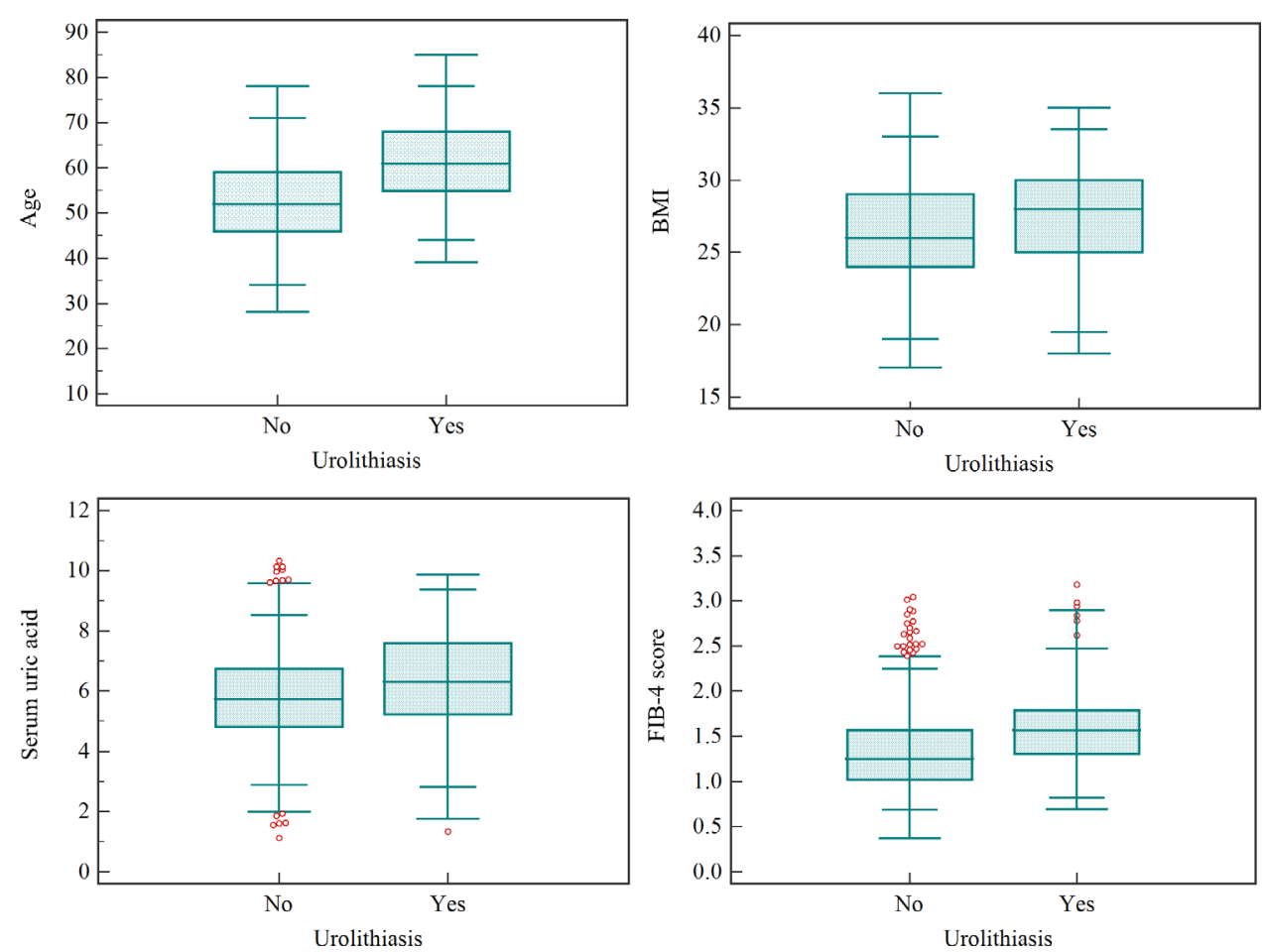

Figure 2 Box-plots of age, BMI, serum uric acid and FIB-4 score among NAFLD patients with and without urolithiasis. The horizontal line inside each box represents the median. The horizontal lines above and below each box encompass the IQR (from 25th to 75th percentile), and the vertical lines from the ends of the box encompass the adjacent values (upper: 75th percentile + 1.5 times the IQR, lower: 25th percentile -1.5 times the IQR). BMI, body mass index; NAFLD, non-alcoholic fatty liver disease.

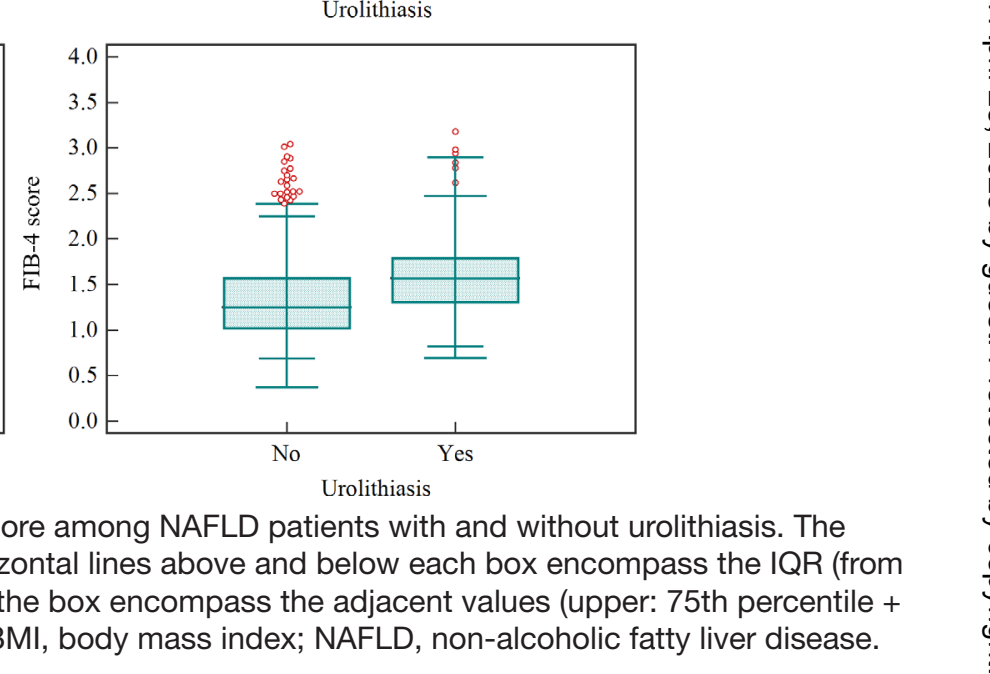

Univariate analysis of associated factors with urolithiasis among NAFLD patients

Patient characteristics, according to the presence of urolithiasis, were shown in table 1 . The median FIB-4 score of the study participants was $1.28(1.04,1.6)$. Univariate analysis showed that urolithiasis patients had a higher FIB-4 score compared with those without urolithiasis $(1.57(1.31,1.78)$ vs $1.25(1.02,1.57)$ ) (table 1 , figure 2). Patients with urolithiasis had a higher median age than those without urolithiasis $(61(55,68)$ vs $52(46$, $59)$ ). The level of serum uric acid was significantly higher in urolithiasis patients than in those without urolithiasis $(6.29 \pm 1.69$ vs $5.75 \pm 1.43, p<0.01)$, with urolithiasis patients having a higher prevalence of hyperuricemia compared with those without urolithiasis $(74 \%$ vs $17 \%, \mathrm{p}<0.01)$. Also, obesity occurred more frequently in urolithiasis patients than in those without urolithiasis $(59 \%$ vs $36 \%$, $\mathrm{p}<0.01$ ), with urolithiasis patients having a higher value of BMI over those without urolithiasis $(27.43 \pm 3.51$ vs $26.14 \pm 3.7, \mathrm{p}<0.01)$. No significant differences existed between groups in the prevalence of diabetes mellitus, hypertension and cardiovascular disease. The percentage of dyslipidemia between the two groups also was similar.

\section{Multivariate analysis of the association between urolithiasis and FIB-4 score as well as other associated factors among NAFLD patients}

The multivariate logistic analysis was performed to control for potential confounders. When adjusting with all potential confounding factors, the logistic model showed 
Table 2 Multivariate logistic regression analysis of factors associated with urolithiasis among NAFLD patents

\begin{tabular}{|c|c|c|c|c|}
\hline \multirow[b]{2}{*}{ Variables } & \multirow[b]{2}{*}{ Comparison } & \multicolumn{3}{|c|}{ Logistic model on the risk of urolithiasis } \\
\hline & & OR & $95 \% \mathrm{Cl}$ for OR & $P$ value \\
\hline Age & Per unit increase & 1.11 & 1.08 to 1.13 & $<0.01$ \\
\hline Hyperuricemia & Yes versus no & 3.79 & 2.67 to 5.36 & $<0.01$ \\
\hline FIB-4 score & Per unit increase & 1.58 & 1.06 to 2.31 & $<0.05$ \\
\hline
\end{tabular}

NAFLD, non-alcoholic fatty liver disease.

four independent factors associated with urolithiasis, including FIB-4 score (OR=1.58; 95\% CI 1.06 to 2.31), age $(\mathrm{OR}=1.11 ; 95 \%$ CI 1.08 to 1.13$)$, obesity $(\mathrm{OR}=3.16$; $95 \% \mathrm{CI} 2.29$ to 4.39 ) and hyperuricemia ( $\mathrm{OR}=3.79$; $95 \%$ CI 2.67 to 5.36 ), suggesting an independent association between FIB-4 score and urolithiasis among individuals with NAFLD (table 2). Accordingly, the multivariate logistic model was based on the algorithm as follows: $\mathrm{c}=0.1$ *age $+1.15^{*}$ obesity $(1$ or 0$)+1.33^{*}$ hyperuricemia $(1$ or 0$)+1.58 *$ FIB- 4 score-11.96. Although ALT differed between those with or without urolithiasis, the multivariate analysis failed to show an independent association between ALT and prevalent urolithiasis among subjects with NAFLD.

\section{Diagnostic value of FIB-4 score and the multivariate model in the detection of prevalent urolithiasis among patients with NAFLD}

ROC analysis was used to assess the diagnostic value of FIB-4 score as well as other associated factors for identifying urolithiasis among NAFLD patients. The AUROC of age, BMI, the value of serum uric acid and FIB-4 score for identifying urolithiasis was 0.749 (95\% CI 0.729 to 0.767 ), 0.612 (95\% CI 0.591 to 0.633$), 0.602$ (95\% CI 0.580 to 0.623 ) and 0.686 (95\% CI 0.665 to 0.706$)$, respectively (table 3, figure 3). The diagnostic accuracy of the multivariate model (FIB-4 score, age, obesity, hyperuricemia) for identifying urolithiasis (AUROC $=0.813$, 95\% CI 0.795 to 0.829 ) was significantly higher than that of a single use of age, BMI, the value of serum uric acid and FIB-4 score $(p<0.001)$ (figure 4$)$. By maximising the sum of sensitivity + specificity, the optimal cut-off value of $>-2.23$ for the multivariate model provides a sensitivity of $76 \%$ and a specificity of $74 \%$ for predicting urolithiasis among NAFLD patients.

\section{DISCUSSION}

This study demonstrated that the FIB-4 score, a surrogate marker of liver fibrosis, is associated with urolithiasis among NAFLD patients. This association persisted after adjustment for potential metabolic factors. A logistic regression model identified four independent factors associated with urolithiasis: FIB- 4 score (OR=1.58; $95 \%$ CI 1.06 to 2.31$)$, age $(\mathrm{OR}=1.11 ; 95 \%$ CI 1.08 to 1.13$)$, obesity
$(\mathrm{OR}=3.16 ; 95 \% \mathrm{CI} 2.29$ to 4.39$)$ and hyperuricemia $(\mathrm{OR}=3.79 ; 95 \%$ CI 2.67 to 5.36$)$.

Several cross-sectional and cohort studies have argued that there is a potential relationship between ultrasonography-diagnosed NAFLD and an increased risk of urinary calculi, independently of established risk factors. ${ }^{21-24}$ The findings of the present study were similar to a cross-sectional study involving a total of 3719 Chinese men, showing that NAFLD was related to a higher prevalence of urinary calculi, independently of age, education status, smoking habit, alcohol consumption, physical activity and BMI. The association of NAFLD with prevalent urolithiasis has also been documented in a large cohort study involving 208578 Korean adults who underwent a health check-up examination between January 2002 and December 2014, indicating that the presence of NAFLD was significantly linked to an increased prevalence of urolithiasis. ${ }^{25}$ The studies mentioned above, however, have not yet determined the impact of hepatic fibrosis on urolithiasis among NAFLD patients. The result of our research extended the findings in preliminary studies,

Table 3 Comparison of the diagnostic value of FIB-4 score and other associated factors for the identification of urolithiasis among NAFLD patients

\section{Urolithiasis in patients with NAFLD}

\begin{tabular}{lll}
\cline { 2 - 3 } Variable & AUROC & $\mathbf{9 5 \%} \mathbf{C l}$ \\
\hline Age & 0.749 & 0.729 to 0.767 \\
BMI & 0.612 & 0.591 to 0.633 \\
\hline Serum uric acid & 0.602 & 0.580 to 0.623 \\
FIB-4 score & 0.686 & 0.665 to 0.706 \\
\hline The multivariate model & 0.813 & 0.795 to 0.829 \\
\hline
\end{tabular}

\begin{tabular}{lc}
\hline Comparison of AUROC & P value \\
\hline Age versus the multivariate model & $<0.001$ \\
Serum uric acid versus the multivariate model & $<0.001$ \\
BMI versus the multivariate model & $<0.001$ \\
FIB-4 score versus the multivariate model & $<0.001$ \\
\hline
\end{tabular}

AUROC, area under a ROC curve; BMI, body mass index; NAFLD, non-alcoholic fatty liver disease. 

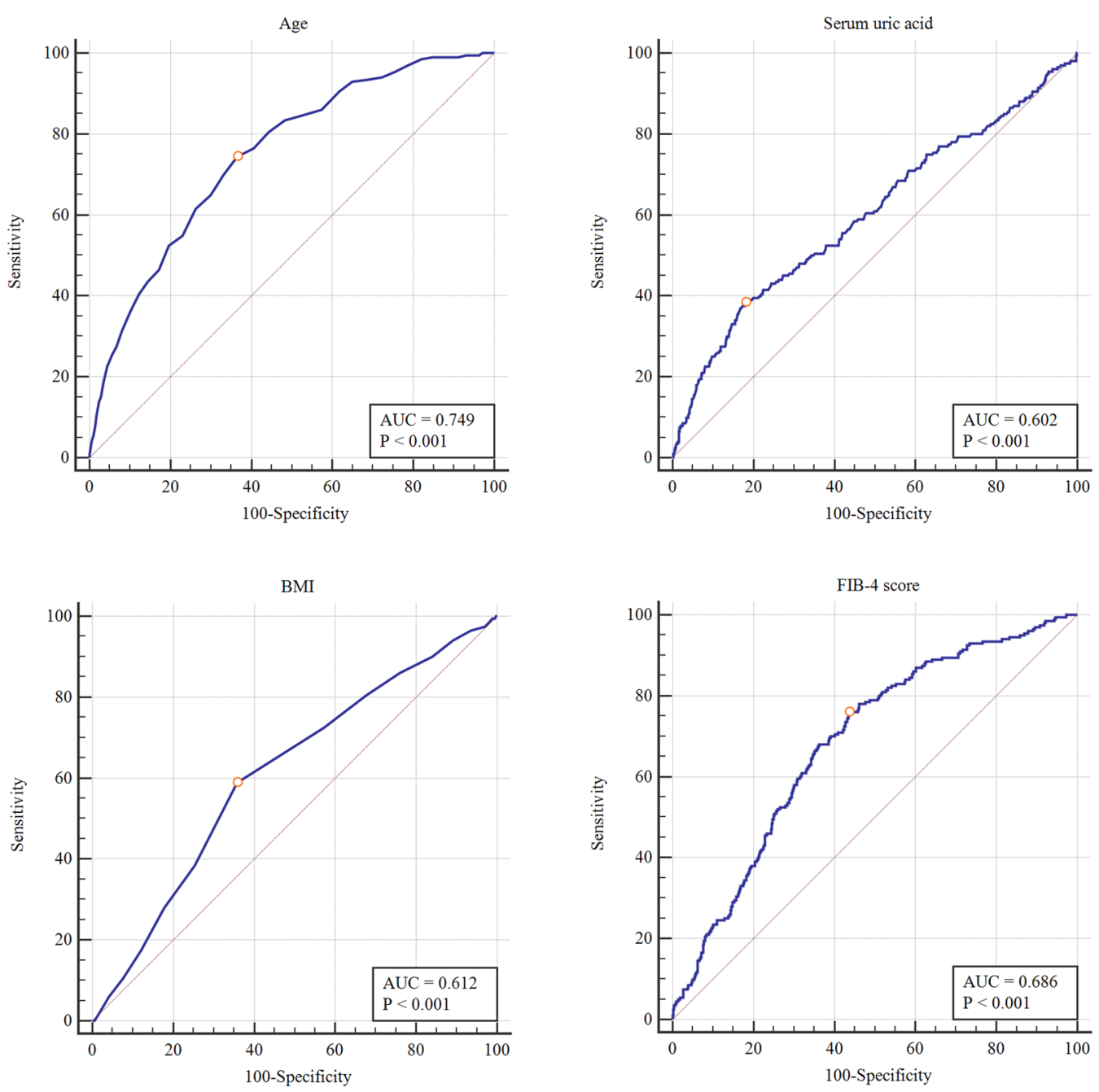

Figure 3 ROC curves of age, BMI, serum uric acid and FIB-4 score for the identification of urolithiasis among NAFLD patients. The diagonal line represents detection achieved by chance alone (AUROC $=0.50$ ); the ideal $A U R O C$ is 1.00 . AUROC, area under a ROC curve; AUC, area under the curve; BMI, body mass index; NAFLD, non-alcoholic fatty liver disease; ROC, receiver operating characteristic.

demonstrating that urolithiasis was linked to a higher value of non-invasive hepatic fibrosis score.

Our findings suggested that the FIB-4 score was associated with urolithiasis, although FIB-4 score was used as a surrogate marker for hepatic fibrosis. These findings suggested that the FIB-4 score might severe as a tool to provide additional risk stratification for identifying urolithiasis among NAFLD patients. We furtherly assessed the diagnostic value of FIB-4 score as well as other associated factors in the detection of urolithiasis among patients with NAFLD. Age, BMI, the value of serum uric acid and FIB-4 score provide AUROCs of 0.749 (95\% CI 0.729 to $0.767), 0.612$ (95\% CI 0.591 to 0.633$), 0.602$ (95\% CI 0.580 to 0.623 ) and 0.686 (95\% CI 0.665 to 0.706 ) for identifying urolithiasis, respectively (table 3 , figure 3 ). The diagnostic value of the multivariate logistic model (age, obesity, hyperuricemia, FIB-4 score) for identifying urolithiasis was 0.813 (0.795 to 0.829 ) (figure 4). Moreover, the multivariate model had a significantly higher value of AUROC than that of a single use of age, BMI, the value of serum uric acid and FIB- 4 score $(p<0.001)$ (figure 4).

The close intercorrelations between NAFLD, insulin resistance, metabolic syndrome and urolithiasis make it challenging to draw a causal relationship responsible for the increased prevalence of urolithiasis observed in individuals with NAFLD. The underlying mechanisms of our findings are still speculative. Current evidence indicates that NAFLD and urolithiasis share multiple common underlying metabolic factors, such as diabetes, hypertension, obesity and metabolic syndrome, representing the most plausible explanation for the association between NAFLD and urolithiasis. ${ }^{26-29}$ Collectively, future prospective studies addressing this issue are needed to draw a definitive conclusion.

Although the underlying biological mechanism linking NAFLD to urolithiasis is not entirely understood, several plausible mechanisms have been proposed. The role of reactive oxygen species (ROS) production and oxidative stress (OS) development in the kidneys stone formation 


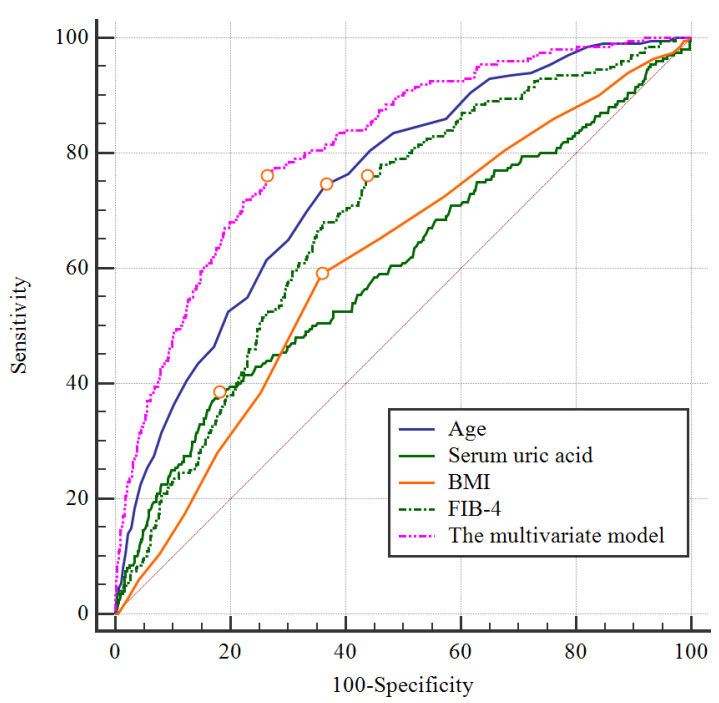

Figure 4 Comparison of ROC curves of age, BMI, serum uric acid, FIB-4 score and the multivariate model (algorithm based on FIB-4 score) for identifying urolithiasis among patients with NAFLD. The diagonal line represents detection achieved by chance alone (AUROC $=0.50)$; the ideal AUROC is 1.00. The diagonal line represents detection achieved by chance alone (AUROC=0.50); the ideal AUROC is 1.00 . AUROC, area under a ROC curve; BMI, body mass index; NAFLD, non-alcoholic fatty liver disease; ROC, receiver operating characteristic.

have attracted considerable scientific interests. ROS could promote the production of crystallisation inhibitors, leading to a decreased incidence of stone formation. ${ }^{30-32}$ However, reduced antioxidant capacity in patients with metabolic syndrome results in increased crystallisation, subsequently contributing to OS and urolithiasis. Also, antioxidants and inhibitors of ROS generating enzymes could decrease renal calcium oxalate $(\mathrm{CaOx})$ crystal deposition, suggesting a critical involvement of ROS in the pathogenesis of urolithiasis. ${ }^{3133}$ Moreover, metabolic syndrome, which has been shown to alter urinary constituents, contributes to an increased risk of both uric acid and $\mathrm{CaOx}$ stone formation. ${ }^{34-36}$ Urolithiasis, particularly in NAFLD patients, has been regarded as a component of the metabolic syndrome in terms of the metabolic factors increasingly involved in the pathophysiology of urolithiasis. $^{37}$

This study has several limitations to be considered in the interpretation of our findings. First, the present study was performed at a single centre. Moreover, the multivariate model for the detection of urolithiasis has limited generalisability because it was not validated in an external cohort. Second, the causality of the link between the non-invasive hepatic fibrosis score of NAFLD and urolithiasis could not be established, as the cross-sectional design of this study. Third, the NAFLD was diagnosed with ultrasonography, which has high sensitivity in establishing the presence of fatty liver but is subject to measurement error. Again, although renal ultrasound remains an effective means for detecting renal stones, it has limitations in identifying small stones and ureteral calculus. Fourth, hepatic fibrosis among NAFLD patients was evaluated with FIB-4 score, a non-invasive maker, but not confirmed by liver biopsy. It is, however, not justified to perform a liver biopsy in all participants with NAFLD.

Despite these limitations, this study has multiple strengths. First, a one-gate design was adopted with all included subjects being diagnosed as NAFLD, thus avoiding selection bias in this study. Second, detailed information on multiple metabolic parameters was available. Multiple potential confounding factors were adjusted in the logistical regression model to determine factors associated with urolithiasis among NAFLD patients. Moreover, after adjustment for confounders, the positive correlation between the FIB-4 score and urolithiasis persistently existed. This association might suggest that urolithiasis is not merely a marker of the shared metabolic factors but urolithiasis itself as a consequence of NAFLD.

In conclusion, our results suggest an association between the non-invasive hepatic fibrosis score and urolithiasis among NAFLD patients. Further, a novel algorithm based on FIB-4 score could serve as a useful tool for identifying individuals with a higher risk of urolithiasis among NAFLD patients. Patients with NAFLD should be carefully monitored for urolithiasis, although future experimental and large-scale cohort studies are needed to elucidate the underlying biological mechanisms and determine whether interventions improving NAFLD can also decrease the risk of urolithiasis.

Contributors SQ and SW conceived and designed the study, carried out the statistical analysis and wrote the manuscript. JW, $\mathrm{CZ}$ and $\mathrm{YZ}$ helped design the study. YX and XW contributed to data analysis.

Funding This study is funded by the Science and Technology Department of Jilin Province (Grant No. 20160414036GH), the Education Department of Jilin Province (Grant No. 2015-517), the Development and Reform Commission of Jilin Province (2015y031-2) and the Wu Jieping Medical Foundation (320.6750.14028).

Competing interests None declared.

Patient consent for publication Not required.

Ethics approval This study was approved by the Ethics Committee of China-Japan Hospital of Jilin University.

Provenance and peer review Not commissioned; externally peer reviewed. Data availability statement No data are available.

Open access This is an open access article distributed in accordance with the Creative Commons Attribution Non Commercial (CC BY-NC 4.0) license, which permits others to distribute, remix, adapt, build upon this work non-commercially, and license their derivative works on different terms, provided the original work is properly cited, appropriate credit is given, any changes made indicated, and the use is non-commercial. See: http://creativecommons.org/licenses/by-nc/4.0/.

\section{REFERENCES}

1. Adams LA, Lymp JF, St. Sauver J, et al. The natural history of nonalcoholic fatty liver disease: a population-based cohort study. Gastroenterology 2005;129:113-21.

2. Angulo P. Nonalcoholic fatty liver disease. N Engl J Med Overseas Ed 2002;346:1221-31.

3. Farrell GC, Larter CZ. Nonalcoholic fatty liver disease: from steatosis to cirrhosis. Hepatology 2006;43:S99-112.

4. Marchesini Get al. Nonalcoholic fatty liver, steatohepatitis, and the metabolic syndrome. Hepatology 2003;37:917-23. 
5. Dietrich P, Hellerbrand C. Non-Alcoholic fatty liver disease, obesity and the metabolic syndrome. Best Pract Res Clin Gastroenterol 2014;28:637-53.

6. Wong YV, Cook P, Somani BK. The association of metabolic syndrome and urolithiasis. Int J Endocrinol 2015;2015:570674.

7. Agrawal V, Shah A, Rice C, et al. Impact of treating the metabolic syndrome on chronic kidney disease. Nat Rev Nephrol 2009;5:520-8.

8. Chen Jet al. Insulin resistance and risk of chronic kidney disease in nondiabetic US adults. J Am Soc Nephro 2003;14:469-77.

9. Machado MV, Gonçalves S, Carepa F, et al. Impaired renal function in morbid obese patients with nonalcoholic fatty liver disease. Liver Int 2012;32:241-8

10. Park CW, Tsai NT, Wong LL. Implications of worse renal dysfunction and medical comorbidities in patients with NASH undergoing liver transplant evaluation: impact on MELD and more. Clin Transplant 2011;25:E606-11.

11. Yilmaz $\mathrm{Y}$, Alahdab $\mathrm{YO}$, Yonal $\mathrm{O}$, et al. Microalbuminuria in nondiabetic patients with nonalcoholic fatty liver disease: association with liver fibrosis. Metabolism 2010;59:1327-30.

12. Targher G, Bertolini L, Rodella S, et al. Relationship between kidney function and liver histology in subjects with nonalcoholic steatohepatitis. CJASN 2010;5:2166-71.

13. HW X, Hsu YC, Chang $\mathrm{CH}$, et al. High FIB-4 index as an independent risk factor of prevalent chronic kidney disease in patients with nonalcoholic fatty liver disease. Hepatol Int 2016;10:340-6.

14. Qin S, Wang S, Wang X, et al. Non-Alcoholic fatty liver disease and the risk of urolithiasis: a systematic review and meta-analysis. Medicine 2018;97:e12092.

15. McPherson S, Stewart SF, Henderson E, et al. Simple non-invasive fibrosis scoring systems can reliably exclude advanced fibrosis in patients with non-alcoholic fatty liver disease. Gut 2010;59:1265-9.

16. Chalasani N, Younossi Z, Lavine JE, et al. The diagnosis and management of non-alcoholic fatty liver disease: practice guideline by the American association for the study of liver diseases, American College of gastroenterology, and the American gastroenterological association. Hepatology 2012;55:2005-23.

17. Pearle MS, Goldfarb DS, Assimos DG, et al. Medical management of kidney stones: AUA guideline. J Urol 2014;192:316-24.

18. Organization WH. The Asia-Pacific perspective: redefining obesity and its treatment. Sydney: Health Communications Australia, 2000

19. DeLong ER, DeLong DM, Clarke-Pearson DL. Comparing the areas under two or more correlated receiver operating characteristic curves: a nonparametric approach. Biometrics 1988;44:837-45.

20. Zhang Z, Gayle AA, Wang J, et al. Comparing baseline characteristics between groups: an introduction to the CBCgrps package. Ann Transl Med 2017;5.

21. Einollahi B, Naghii MR, Sepandi M. Association of nonalcoholic fatty liver disease (NAFLD) with urolithiasis. Endocr Regul 2013;47:27-32.
22. Nam IC, Yoon JH, Park SH, et al. Association of non-alcoholic fatty liver disease with renal stone disease detected on computed tomography. European Journal of Radiology Open 2016;3:195-9.

23. Zeina A-R, Goldenberg L, Nachtigal A, et al. Association between nephrolithiasis and fatty liver detected on non-enhanced CT for clinically suspected renal colic. Clin Imaging 2017;43:148-52.

24. Wei Y-P, Lin X-G, He R-Q, et al. Epidemiologic association of nonalcoholic fatty liver disease and urinary calculi: a populationbased cross-sectional study in southern China. Iran J Kidney Dis 2018;12:112-9.

25. Kim S, Chang Y, Sung E, et al. Non-Alcoholic fatty liver disease and the development of nephrolithiasis: a cohort study. PLoS One 2017;12:e0184506.

26. Besiroglu H, Otunctemur A, Ozbek E. The metabolic syndrome and urolithiasis: a systematic review and meta-analysis. Ren Fail 2015;37:1-6.

27. Rendina D, De Filippo G, D’Elia L, D'Elia L, et al. Metabolic syndrome and nephrolithiasis: a systematic review and meta-analysis of the scientific evidence. J Nephrol 2014;37:371-6.

28. Spatola L, Ferraro PM, Gambaro G, Leonardo S, Pietro FM, Giovann $\mathrm{G}$, et al. Metabolic syndrome and uric acid nephrolithiasis: insulin resistance in focus. Metabolism 2018;83:225-33.

29. Spatola L, Angelini C, Badalamenti S, et al. Kidney stones diseases and glycaemic statuses: focus on the latest clinical evidences. Urolithiasis 2017;45:457-60.

30. Sharma M, Kaur T, Singla SK. Role of mitochondria and NADPH oxidase derived reactive oxygen species in hyperoxaluria induced nephrolithiasis: therapeutic intervention with combinatorial therapy of $\mathrm{N}$-acetyl cysteine and apocynin. Mitochondrion 2016;27:15-24.

31. Khan SR. Reactive oxygen species, inflammation and calcium oxalate nephrolithiasis. Transl Androl Urol 2014;3:256-76.

32. Khan SR. Reactive oxygen species as the molecular modulators of calcium oxalate kidney stone formation: evidence from clinical and experimental investigations. J Urol 2013;189:803-11.

33. Hyuk Hong S, Lee H-J, Jung Sohn E, et al. Anti-nephrolithic potential of resveratrol via inhibition of ROS, MCP-1, hyaluronan and osteopontin in vitro and in vivo. Pharmacological Reports 2013;65:970-9.

34. Polat EC, Ozcan L, Cakir SS, et al. Relationship between calcium stone disease and metabolic syndrome. Urol J 2015;12:2391-5.

35. Rendina D, De Filippo G, Zampa G, et al. Characteristic clinical and biochemical profile of recurrent calcium-oxalate nephrolithiasis in patients with metabolic syndrome. Nephrology Dialysis Transplantation 2011;26:2256-63.

36. Sakhaee K, Capolongo G, Maalouf NM, et al. Metabolic syndrome and the risk of calcium stones. Nephrology Dialysis Transplantation 2012;27:3201-9.

37. Kohjimoto Y, Iba A, Sasaki Y, et al. [Metabolic syndrome and nephrolithiasis]. Hinyokika Kiyo 2011;57:43-7. 